\title{
A LOCAL SPECTRAL THEORY FOR OPERATORS. II
}

\author{
BY J. G. STAMPFLI ${ }^{1}$
}

Communicated by Henry Helson, November 22, 1968

I. Introduction. Let $T$ be an operator on a Banach space $B$. Let $\sigma(T)$, the spectrum of $T$, lie on a line, a circle, or, more generally, a smooth curve. If the resolvent $R_{z}(T)=(T-z I)^{-1}$ satisfies a growth condition with respect to $\sigma(T)$, it is possible, in many cases, to develop an invariant subspace decomposition for $T$. We mention explicitly the work of Bartle [1], Godement [4], Leaf [6], Lorch [7], Schwartz [13], Wermer [19], and Wolf [20]. Since, in the references cited, none of the subspaces is necessarily complemented by an invariant subspace, one can not expect this invariant subspace decomposition to generate a countably additive resolution of the identity. Such a spectral resolution is precisely the achievement of the Dunford theory [3], but there it was necessary to assume a second condition in order to obtain it. This condition (Dunford Boundedness) is not easy to verify in practice.

In this note, we will study several situations in Hilbert space, where a strong growth condition on the resolvent is sufficient to guarantee a countably additive resolution of the identity, i.e., the operator turns out to be similar to a normal operator. The results in $\$ 3$ generalize, and are dependent on, some recent work of Gokhberg and Krein. We will only sketch proofs. Complete details will appear in [16] and elsewhere.

From now on, the underlying space is always a Hilbert space. All operators are bounded. By a smooth Jordan curve, we mean a Jordan curve of class $C^{2}$ (in the complex plane).

II. In this section we study conditions on the resolvent which insure normality.

Lemma 1. Let $\left\|(T-\lambda)^{-1}\right\| \leqq 1 / d$ where $0<d<|\lambda|$. Then

$$
\left\|\left(T^{-1}-\frac{\bar{\lambda}}{|\lambda|^{2}-d^{2}}\right)^{-1}\right\| \leqq \frac{|\lambda|^{2}-d^{2}}{d} .
$$

THEOREM 1. Let $U$ be an open set and let $\sigma(T) \cap U$ lie in the smooth Jordan curve C. Let $\left\|R_{\lambda}(T)\right\| \leqq 1 /$ dist $[\lambda, C]$ for $\lambda \in U$. Then $T=T_{1} \oplus T_{2}$ where $T_{1}$ is normal, $\sigma\left(T_{1}\right)=$ closure $[\sigma(T) \cap U]$ and $\sigma\left(T_{2}\right) \subset \sigma(T) \cap U^{\prime}$. tion.

1 The author gratefully acknowledges the support of the National Science Founda- 
( $\oplus$ denotes orthogonal direct sum.)

The proof of Theorem 1 is too long to even sketch. It relies heavily on successive application of Lemma 1 coupled with material on the numerical range found in [15].

Corollary 1. Let $\sigma(T)$ lie in the smooth Jordan curve $C$. Let $\left\|R_{\lambda}(T)\right\| \leqq 1 / \operatorname{dist}[\lambda, C]$ for $\lambda$ in a neighborhood of $C$. Then $T$ is normal.

Corollary 1 was proved for $C=$ Reals by Nieminen [10] and $C=$ unit circle by Donoghue [2] with a slightly stronger growth condition.

Since $\left\|R_{\lambda}(T)\right\|=1 / \operatorname{dist}[\lambda, \sigma(T)]$ for any hyponormal operator $T$, Theorem 1 is useful in dealing with this class of operators. (See for example the question raised by Putnam in [12]; also Putnam [11] and Stampfli [14].)

Definition. An operator $T$ on a Hilbert space $H$ is in $\mathfrak{C}_{\rho}$ if there exists a Hilbert space $K \supset H$, a constant $\rho>0$, and a unitary operator $U$ on $K$, such that $T^{n}=\rho P U^{n} P$ for $n=1,2, \cdots$, where $P$ is the selfadjoint projection of $K$ on $H$.

CoRollary 2. If $T \in \mathfrak{e}_{\alpha}$ and $T^{-1} \in \mathfrak{e}_{\beta}$, then $T$ is unitary.

III. In this section, we will generalize some recent work of Gokhberg and Krein [5]. First, we state their theorem which depends on a deep result of Nagy and Foiaș [17].

THEOREM (G-K). Let $T$ be a contraction. If $\left\|R_{\lambda}(T)\right\| \leqq K /(1-|\lambda|)$ for $|\lambda|<1$, then $T$ is similar to a unitary operator.

The next lemma is a modest improvement.

LEMMA 2. Let

(i) $\left\|R_{\lambda}(T)\right\| \leqq 1 /(|\lambda|-1)$ for $|\lambda|>1$, and

(ii) $\left\|R_{\lambda}(T)\right\| \leqq K /(1-|\lambda|)$ for $|\lambda|<1$.

Then $T$ is similar to a unitary.

Proof. Condition (i) implies that $W(T)$, the numerical range of $T$, lies in the unit disc (and conversely). Hence, by a result of Nagy and Foias [18], $T=Q A Q^{-1}$ where $A$ is a contraction. But $\left\|R_{\lambda}(A)\right\|$ $\leqq\|Q\|\left\|Q^{-1}\right\| K /(1-|\lambda|)$ for $|\lambda|<1$. Thus, $A$ and hence $T$ are similar to a unitary operator by the previous theorem.

Theorem 2. Let $\sigma(T)$ lie in the unit circle. Let

(i) $\|R(T)\| \leqq K /(1-|\lambda|)$ for $\alpha<|\lambda|<1$, and

(ii) $\|R(T)\| \leqq 1 /(|\lambda|-1)$ for $1<|\lambda|<\beta$.

Then $T$ is similar to a unitary operator. 
Proof. By suitable use of Lemma 1, we can reduce the proof to the case where $T$ satisfies (i) and (ii) in a sector (from 0 to infinity). Let $\delta$ be a small arc of the unit circle, contained in this sector. There exists an invariant subspace $H_{\delta}$ of $T$ such that $\sigma\left(T \mid H_{\delta}\right) \subset \delta$. Moreover, it follows from the Lorch approximation theorem [7], and the growth conditions that $W\left(T \mid H_{\delta}\right)$ is contained in the unit disk. Hence, $T \mid H_{\delta}$ is similar to a unitary by Lemma 2. Unfortunately, it is not clear that there exits a subspace complementary to $H_{\delta}$ which is invariant under $T$. This difficulty can be overcome by cutting $T$ down to a subset of $\delta$ and estimating the angle between appropriate subspaces. Repeating this argument a finite number of times completes the proof.

REMARK. It makes no difference if the roles of $K$ and 1 are interchanged in (i) and (ii). Moreover, (i) and (ii) are not needed for the entire circle, but only in a neighborhood of $\sigma(T)$. In fact, one can even recover a variation of Theorem 1 . Let $U$ be an open set and let $U \cap \sigma(T) \neq \varnothing$ lie in the unit circle. Further, let $R_{\lambda}(T)$ satisfy (i) and (ii) for $\lambda \in U$. Then, $T=T_{1}+T_{2}$, where $T_{1}$ is similar to a normal and $\sigma\left(T_{1}\right)$ can be chosen to be any closed subset of $\sigma(T)$ contained in $U$. $(+$ denotes direct sum, i.e., the underlying spaces are complementary.)

Theorem 1 can be used to obtain results on operators with real spectrum.

Corollary 1. Let $\sigma(T)$ be real. Let

(i) $\left\|R_{\lambda}(T)\right\| \leqq 1 / \operatorname{Im} \lambda$ for $0<\operatorname{Im} \lambda<\alpha$, and

(ii) $\left\|R_{\lambda}(T)\right\| \leqq K /|\operatorname{Im} \lambda|$ for $\beta<\operatorname{Im} \lambda<0$.

Then $T$ is similar to a selfadjoint operator.

Since growth conditions on the resolvent are usually applied near the spectrum or at infinity, the next corollary, at first glance, seems surprising.

Corollary 2. Let $\sigma(T)$ be real. Let $\left\|R_{\lambda}(T)\right\| \leqq 1 / \operatorname{Im} \lambda$ for $\lambda$ in a neighborhood of the parabola $2 y=x^{2}+1(z=x+i y)$. If $\left\|R_{\lambda}(T)\right\| \leqq$ $1 /|\operatorname{Im} \lambda|$ for $\lambda$ in a neighborhood of $2 y=-\left(x^{2}+1\right)$ then $T$ is selfadjoint. If $\left\|R_{\lambda}(T)\right\| \leqq K /|\operatorname{Im} \lambda|$ for $\beta<\operatorname{Im} \lambda<0$, then $T$ is similar to a selfadjoint operator.

Corollaries 1 and 2 follow easily by taking the Cayley transform of $T$. The remark following Theorem 2 applies here as well.

IV. The growth condition on the resolvent in the preceding section can not be substantially weakened as seen by the following 
Example (McCarthy and Schwartz [9], A. S. Markus [8]). There exists an operator $T$ on Hilbert space, such that the spectrum of $T$ is real, and $\left\|R_{\lambda}(T)\right\| \leqq K /|\operatorname{Im} \lambda|$ for all $\lambda$. However, $T$ is not similar to a selfadjoint operator.

\section{REFERENCES}

1. R. G. Bartle, Spectral localization of operators in Banach space, Math. Ann. 153 (1964), 261-269.

2. W. F. Donoghue, On a problem of Nieminen, Inst. Hautes Etudes Sci. Publ. Math. 16 (1963), 127-129.

3. N. Dunford, $A$ survey of the theory of spectral operators, Bull. Amer. Math. Soc. 64 (1958), 217-274.

4. R. Godement, Théorèmes Tauberiens et théorie spectral, Ann. Sci. École Norm. Sup. 64 (1947), 119-138.

5. I. C. Gokhberg and M. G. Krein, On a description of contraction operators similar to unitary operators, Funkcional. Anal. i Priložen. 1 (1967), 38-60= Functional Anal. Appl. 1 (1967), 33-52.

6. G. Leaf, A spectral theory for a class of linear operators, Pacific J. Math. 13 (1963), 141-155.

7. E. R. Lorch, The integral representation of weakly almost periodic transformations in reflexive vector spaces, Trans. Amer. Math. Soc. 49 (1941), 18-40.

8. A. S. Markus, Some criteria for the completeness of a system of root vectors of a linear operator in a Banach space, Mat. Sb. 70 (112) (1966), 526-561; English transl. Amer. Math. Soc. Transl. (2) 85 (1969).

9. C. A. McCarthy and J. Schwartz, On the norm of a finite Boolean algebra of projections, and applications to theorems of Kreiss and Morton, Comm. Pure Appl. Math. 18 (1965), 191-201.

10. T. Nieminen, $A$ condition for the self-adjointness of an operator, Ann. Acad. Sci. Fenn. Ser. A I 316 (1962), 3-5.

11. C. R. Putnam, On the spectra of seminormal operators, Trans. Amer. Math. Soc. 119 (1965), 509-523.

12. - Hyponormal operators and low density spectra, Math. Z. 106 (1968), 167-169.

13. J. Schwartz, Subdiagonalization of operators in Hilbert space with compact imaginary part, Comm. Pure Appl. Math. 15 (1962), 159-172.

14. J. G. Stampfli, Hyponormal operators and spectral density, Trans. Amer. Math. Soc. 117 (1965), 469-476.

15. - Minimal range theorems for operators with thin spectra, Pacific J. Math. 23 (1967), 601-612.

16. - A local spectral theory for operators, J. Functional Analysis (to appear).

17. B. Sz. Nagy and C. Foiaş, Sur les contractions de l'espace de Hilbert. X; Contractions similaires d des transformations unitaires, Acta Sci. Math. 26 (1965), 79-91.

18. - Similitude des operateurs de classe $\mathfrak{C}_{\rho} d$ des contractions, C. R. Acad. Sci. Paris, Ser. A 264 (1967), 1063-1065. 622.

19. J. Wermer, The existence of invariant subspaces, Duke Math. J. 19 (1952), 615-

20. F. Wolf, Operators in Banach space which admit a generalized spectral decomposition, Nederl. Akad. Wetensch. Proc. Ser. A 60 =Indag. Math. 19 (1957), 302-311.

Indiana University, Bloomington, Indiana 47405 